\title{
Inhibitory interneurons in Alzheimer’s disease
}

\author{
Vargova $\mathrm{G}^{1}$, Vogels $\mathrm{T}^{1}$, Kostecka $\mathrm{Z}^{2}$, Hromadka $\mathrm{T}^{1}$ \\ Institute of Neuroimmunology, Slovak Academy of Sciences, Bratislava, Slovakia. tomas.hromadka@savba.sk
}

\begin{abstract}
Alzheimer's disease is currently the most common neurodegenerative disorder, characterized by distinct cognitive and sensory deficits. The underlying pathogenetic mechanisms, however, still remain elusive. How the molecular and morphological changes associated with Alzheimer's disease affect information processing in neuronal circuits and translate into cognitive dysfunction is unclear. Inhibitory interneurons have recently emerged as one of the earliest and important culprits in mediating dysfunction of neuronal circuits in neurodegeneration. Amyloid-beta and tau protein have been both linked to interneuron dysfunction, and likely play an important, albeit unknown, role in mediating changes in the overall activity of neuronal circuits. Resolving the role of inhibitory interneurons in neurodegeneration-specific changes in neuronal activity is crucial for understanding the impact of Alzheimer's disease on brain function and even for possible identification of effective treatments and diagnostic techniques (Ref. 63). Text in PDF www.elis.sk.

KEY WORDS: Alzheimer's disease, interneurons, in-vivo, cortical circuits, mouse models.
\end{abstract}

\section{Alzheimer's disease}

Alzheimer's disease (AD) is currently the most prevalent neurodegenerative disorder and represents the most common cause of dementia, accounting for estimated $50-70 \%$ of all cases, and affecting approximately 30 million people worldwide. AD is a systemic disease characterized by distinct progressive cognitive deficits including disturbances in memory, language, executive function, and sensory processing $(1,2)$. Alzheimer's disease develops over many years, even decades. The symptomatic stage of the disease follows a long presymptomatic stage during which pathogenic mechanisms are already at work $(3,4)$. The variableduration presymptomatic stage eventually evolves into prodromal stage characterized by mild cognitive impairment. The final symptomatic stage of the disease then represents a culmination of many years of subtle, perhaps irreversible alterations in brain structure and function. Preclinical stages represent a major challenge for diagnostic procedures aimed at detecting and preventing Alzheimer's disease.

Neurofibrillary tangles (NFTs) and neuritic plaques are the two major histopathological features of Alzheimer's disease. Neurofibrillary tangles are intracellular fibres composed of hyperphosphorylated aggregates of the microtubule-associated protein tau, whereas neuritic plaques are extracellular depositions composed

${ }^{1}$ Institute of Neuroimmunology, Slovak Academy of Sciences, Bratislava, Slovakia, and ${ }^{2}$ The University of Veterinary Medicine and Pharmacy in Kosice, Slovakia

Address for correspondence: T. Hromadka, PhD, Institute of Neuroimmunology of SAS, Dubravska cesta 9, SK-845 10 Bratislava, Slovakia. Phone: +421.2.54788100, Fax: +421.2.54774276

Acknowledgements: This work was supported by EU structural funds ITMS 26240220008 and VEGA grant 2/0148/16. of amyloid-beta (Abeta) peptide. Both tangles and plaques eventually lead to progressive synaptic and neuronal loss in cortical and subcortical structures of the brain (5).

The pathological features associated with Alzheimer's disease influence not only the synaptic activity (6), but also play a role in controlling neuronal activity in larger neuronal networks $(7,8)$. Analysis of spontaneous activity of neurons surrounding amyloid plaques in cerebral cortex suggests an existence of pathological foci of localized hyperactivity $(9,10)$. On the other hand, neurons containing NFTs in the visual cortex appear to be well-integrated into their circuits (11). Network level mechanisms of changes in cortical activity in progressing neurodegeneration remain unresolved, yet these are crucial for understanding the impact of neurodegeneration on brain function and even for possible identification of effective treatments and diagnostic techniques in presymptomatic stages of the disease (12). Determining the relationship between pathological changes at the level of molecules, synapses, neurons, circuits, networks, and cognition represents an urgent challenge in understanding neurodegeneration.

The progression of neurodegeneration is associated with complex changes in overall neuronal activity, such as imbalance between excitatory and inhibitory transmission leading to, for example, epileptic seizures (13). AD patients also exhibit increased silent seizures which likely contribute to cognitive deficits (14). Moreover, network hyperexcitability in Alzheimer's disease impairs cognition (15), and altered neuronal networks may, therefore, also contribute to the neurodegenerative process.

\section{Inhibitory interneurons}

The cerebral cortex contains two main types of neurons, excitatory neurons and interneurons. Most neurons (80 \%) embedded in 
cortical neuronal circuits are excitatory pyramidal neurons. These workhorses of the brain have rather uniform anatomical and physiological properties, and form both long-range and local projections.

Inhibitory interneurons, despite forming only about $20 \%$ of all cortical neurons, offer a dizzying cornucopia of various morphological, molecular, and electrophysiogical properties (16), and form mostly local connections. These interneurons use GABA (gamma-aminobutyric acid) as their neurotransmitter and provide a range of important functions, mainly in maintaining the overall balance of excitatory and inhibitory activity (17) in neuronal networks. Modulation of excitability of various neuronal assemblies and networks mediates cognitive processes, and, perhaps unsurprisingly, inhibitory interneurons have been already implicated in a wide range of neurological and psychiatric disorders, including autism, epilepsy, schizophrenia, and others $(18,19)$.

Particularly important are two prominent classes of inhibitory interneurons (20). Parvalbumin-expressing interneurons are the dominant type of cortical interneurons providing powerful somatic inhibition to postsynaptic partners and displaying fast-spiking activity $(21,22)$. Somatostatin-expressing interneurons, on the other hand, provide distal inhibition and display more gradual, delayed responses to stimulation (23).

Dysfunction of GABAergic transmission has recently also emerged as one of the key players in the pathogenesis of network dysfunction in Alzheimer's disease $(24,12)$, with parvalbuminpositive interneurons likely playing a prominent role $(25,26)$. Alterations in network excitability mediated by a reduction in excitatory synaptic connections on parvalbumin-positive interneurons have been recently implicated in human AD brains (27).

It is, however, still unclear which types of interneurons are the most affected by the relentless process of neurodegeneration. Pathological activity and function of distinct neuronal classes will reverberate across neuronal networks and cause an imbalance in the overall activity of cortical networks. Given their central role in maintaining balance in cortical circuits and function of neuronal networks, and likely mediating cognitive processes, it is crucial to understand how inhibitory interneurons are affected in Alzheimer's disease.

\section{Inhibitory interneurons and amyloid beta}

Interest in the role of network excitability in Alzheimer's disease was first fueled by direct observations that amyloid-beta oligomers and plaques were associated with neuronal hyperexcitability in both the hippocampus and the cortex $(9,10,28)$. Amyloid-beta deposition caused progressive deterioration of neuronal tuning in the visual cortex, which was associated with deficits in visual-pattern discrimination. Interestingly, the loss of function only occurred in the hyperactive neurons within the affected network (29). However, not all network functions seem to be degraded to the same extent, some representations may be selectively preserved even in the presence of amyloid beta depositions, possibly reflecting homeostatic network mechanisms at work (30). These observations highlight the importance of a much more detailed understanding of how pathological protein aggregates affect the behavior of neurons in neural networks instead of simply interpreting the presence of protein pathology as a proxy of network dysfunction.

Network effects of amyloid plaques in the hippocampus are well established and plaques were shown to lead to progressive loss of hippocampal place cell function (31). Furthermore, amyloid beta deposition caused impaired synaptic rewiring and synapse loss in hippocampal oriens-lacunosum-moleculare interneurons. The synaptic deficits were detected both at the axon and the dendrites, indicating network disintegration at the input as well as at the output level, and limiting the fear-learning abilities of APP/ PS1 mice (32).

Amyloid beta aggregates also disrupt the long-range cortical connectivity. These disruptions and associated cognitive deficits could be rescued with GABA(A) receptor agonists (24), suggesting an involvement of cortical inhibitory interneurons. Similarly, restoring the function of PV interneurons restored inhibitory synaptic transmission, network activity, and cognitive deficits in amyloidbeta depositing mice (26). Thus, even though interneurons mostly project locally, their dysfunction and downstream effects on excitatory pyramidal cells can have wide-ranging consequences across distant cortical areas. Furthermore, these studies demonstrate that the modulation of network excitability by inhibitory interneurons could rescue some of the networks disruptions without targeting the underlying protein pathology.

The molecular mechanism of amyloid beta-induced network hyperexcitability are not yet resolved and represent a very active research area. For example, dendritic degeneration of CA1 neurons in the hippocampus has been linked to neuronal hyperexcitability in APP/PS1 mice (33), linking structural abnormalities to characteristic neuronal malfunction in Alzheimer's disease. Furthermore, amyloid beta-induced hyper-excitability and toxicity is dependent on the levels of tau protein, suggesting a joint co-pathogenic effect of both protein AD culprits (34). Under pathological conditions, tau enhances targeting of the tyrosine kinase Fyn to the post-synapse, leading to increased formation of NMDA receptor-PSD95 complexes, which underlie the excitotoxic effects of amyloid beta, e.g. increased calcium influx (35). This amyloid beta-induced activation of NDMA receptors induces signaling through the CAMKK2AMPK kinase pathway, which in turn leads to tau phosphorylation and synaptotoxicity (36).

Intriguingly, several studies suggested that restoring the activity of neuronal networks may also ameliorate amyloid-beta neuropathology. For example, optogenetic restoration of slow wave oscillations restored disruption in calcium signaling and stopped further amyloid-beta deposition (37). Additionally, optogenetic induction and restoration of gamma oscillations by stimulating the parvalbumin-positive interneurons in a mouse model of Alzheimer disease induced a microglial transformation, which led to increased phagocytosis of amyloid-beta by microglia, attenuating amyloid-beta pathology (38). Amyloid-beta pathology and network activity mediated by inhibitory interneurons may thus form a mutually destructive bi-directional relationship during the pathogenesis of Alzheimer's disease. 


\section{Inhibitory interneurons and tau}

Tau protein, in its various disguises, is one of the key players in progressive neurodegeneration. During the pathogenesis of neurodegeneration, tau protein becomes increasingly phosphorylated, forms toxic aggregates, and eventually NFTs (39). Neurons containing neurofibrillary tangles exhibit deficits in synaptic integration, slowly leading to neurodegeneration (40). Even prior to tau depositions, however, elevated levels of soluble tau likely contribute to neuronal dysfunction (41). Tau pathology correlates strongly with cognitive deficits, and the presence of tau aggregates has been linked to clinical manifestations mediated by affected areas (42).

A panoply of tau-based mouse models of neurodegeneration has been used to uncover the complex relationship of tau pathology and activity of neuronal networks in vivo. Even early-stage tau pathology has been shown to be associated with altered network activity (43) and neuronal hypo-excitability (44). Recordings in freely moving rTg4510 mice identified synaptic, neuronal, and network deficits related to aberrant behavior in response to tau pathology $(45,46,47)$. Electrophysiological changes are observed early in rTg4510 and precede tau-mediated morphological changes in neurons, suggesting that pathological tau protein variants can influence neuronal activity even before the appearance of NFTs (50). Furthermore, tau pathology has been linked to the loss of excitatory neurons in the hippocampus, increase in interneuron activity, grid cell dysfunction and associated spatial memory deficits in mice expressing tau-P301L in the entorhinal cortex (53).

Although tau pathology has been linked to a variety of network deficits, its impact on activity of single neurons is surprisingly modest. Few differences were observed when comparing neurons with and without neurofibrillary tangles (48, 49, 50). Curiously, even neurons with NFTs were observed to have normal baseline calcium signaling, receptive fields, and thus were likely still functionally integrated into their cortical circuits in the visual cortex (11). Severe NFT pathology also did not influence neuronal immediate early gene expression in response to visual stimuli (52). Despite severe tau pathology and widespread synapse loss, neurons in rTg4510 mice still displayed no changes in their calcium signaling (51), suggesting that tau-related neuronal dysfunction is mediated by pathways distinct from those mediating amyloidbeta-related neuronal dysfunction. The Tau-induced neuronal network dysfunction thus seems to be a complex phenomenon which depends on the stage of tau pathology, anatomical area affected, and corresponding compensatory network mechanisms.

Similarly, there seems to be an uneasy relationship between tau pathology and activity of inhibitory interneurons. Tau pathology has been linked to altered synaptic plasticity and increase in interneuron firing in the entorhinal cortex in mice harboring the P301L tau mutation (53). However, the same mutation induced a loss of GABAergic interneurons in the hippocampus, altered synaptic plasticity, and related cognitive deficits (54). Both the synaptic and cognitive deficits could be rescued with a GABA(A) receptor agonist further demonstrating the importance of inhibitory inter- neurons in tau-related cognitive symptoms. Artificially induced tau pathology on P301L background did not change the amount of parvalbumin-positive interneurons, but led to changes in neuronal oscillations and functional uncoupling between the prefrontal cortex and hippocampus. Tau pathology has been strongly linked to general synapse loss in Alzheimer's disease (56) - the strongest correlate of cognitive decline (57) - but it is currently unclear if excitatory or inhibitory synapses are predominantly affected.

Tau pathology in Alzheimer's disease progresses through neuronal networks in a stereotypical pattern (58), most likely spreading throughout the brain along neuronal connections. The activity of neuronal networks themselves can influence the spreading of tau pathology. An optogenetically induced increase in neuronal activity led to faster tau propagation (59). Amyloid-beta also leads to faster tau spreading, and amyloid-beta-induced network hyperaxcitability may be one of underlying mechanisms (60). Indeed, the progression of tau pathology to the medial temporal lobe was only observed in the presence of amyloid pathology in $\mathrm{AD}$ patients (62). Normalizing network excitability (e.g. by targeting inhibitory interneurons) may decrease the spreading of tau pathology throughout the brain. On the other hand, increased synaptic activity may lead to increased clearance of tau pathology by activating the autophagic-lysosomal degradation in synapses (63). Determining the specific impact of tau pathology on the activity and function of inhibitory interneurons remains one of the important questions in the contemporary research of neurodegenerative disorders.

\section{Conclusion}

The focus of research of Alzheimer's disease is changing. From studying molecular changes and cognitive deficits alone, the focus is shifting towards understanding dysfunction of neuronal circuits and networks. How the distinct molecular changes impact the function of various neuronal classes and how these functional changes propagate further into characteristic changes in cognition and behavior are two of the fundamental questions not only in neurodegeneration research, but in neuroscience in general.

Dysfunction of neuronal networks plays a dominant role in the pathophysiology of Alzheimer's disease and neurodegeneration. Tau protein and amyloid-beta, the two typical representatives of AD neuropathology, influence the activity of synapses, neurons, and networks. The activity of neuronal networks can in turn have a profound impact on tau or amyloid-beta-related pathology.

Inhibitory interneurons are in the spotlight of neurodegenerative changes. Given their distinct role in information processing in neuronal circuits, interneurons represent the primary focus in research in 'systems neurodegeneration'. A precise understanding of specific changes in the activity and function of inhibitory interneurons would bring improved understanding of cognitive deficits in Alzheimer's disease. A specific electrophysiological signature of interneuronal activity in neurodegenerative disorders would then provide a new way of testing the efficacy of preventive and therapeutic agents in stopping or even reversing the progression of neurodegeneration and thus would be of great interest to both clinical and basic neuroscience (61). 


\section{References}

1. Brewer AA, Barton B, Dumoulin SO. Visual cortex in aging and Alzheimer's disease: changes in visual field maps and population receptive fields. Front Psychol 2014; 5: 74.

2. Yankner BA, Lu T, Loerch P. The aging brain. Ann Rev Pathol 2008; 3: 41-66.

3. Braak H, Braak E. Staging of Alzheimer-related cortical destruction. Internat Psychogeriat 1997; 9 (Suppl 1): 257-272.

4. Mayeux R. Early Alzheimer's Disease. New Engl J Med 2010; 362 (23): 2194-2201.

5. Serrano-pozo A et al. Neuropathological Alterations in Alzheimer Disease. Cold Spring Harb Perspect Med 2010; 1: a006189.

6. William CM et al. Synaptic plasticity defect following visual deprivation in Alzheimer's disease model transgenic mice. J Neurosci 2012; 32 (23): 8004-8011.

7. Busche MA et al. Rescue of long-range circuit dysfunction in Alzheimer’s disease models. Nature Neurosci 2015; 18 (11): 1623-1630.

8. Palop JJ et al. Article Aberrant Excitatory Neuronal Activity and Compensatory Remodeling of Inhibitory Hippocampal Circuits in Mouse Models of Alzheimer's Disease. Neuron 2007; 55: 697-711.

9. Busche MA et al. Clusters of hyperactive neurons near amyloid plaques in a mouse model of Alzheimer's disease. Science 2008; 321 (5896): 16861689.

10. Kuchibhotla KV et al. Synchronous hyperactivity and intercellular calcium waves in astrocytes in Alzheimer mice. Science 2009; 323 (5918): 1211-1215.

11. Kuchibhotla KV et al. Neurofibrillary tangle-bearing neurons are functionally integrated in cortical circuits in vivo. Proc Nat Acad Sci 2014; 111 (1): 2-6.

12. Palop JJ, Mucke L. Amyloid-beta-induced neuronal dysfunction in Alzheimer's disease: from synapses toward neural networks. Nature Neurosci 2010; 13 (7): 812-818.

13. Vossel KA et al. Seizures and epileptiform activity in the early stages of Alzheimer disease. JAMA Neurology 2013; 70 (9): 1158-1166.

14. Lam AD et al. Silent hippocampal seizures and spikes identified by foramen ovale electrodes in Alzheimer's disease. Nature Med 2017; 23 (6): 678-680.

15. You JC et al. Epigenetic suppression of hippocampal calbindin-D28k by DeltaFosB drives seizure-related cognitive deficits. Nature Med 2017; 23 (11): 1377-1383.

16. Markram $\mathbf{H}$ et al. Interneurons of the neocortical inhibitory system. Nature Rev Neurosci 2004; 5 (10): 793-807.

17. Wehr M, Zador A. Balanced inhibition underlies tuning and sharpens spike timing in auditory cortex. Nature 2003; 426: 442-446.

18. Levitt P, Eagleson KL, Powell EM. Regulation of neocortical interneuron development and the implications for neurodevelopmental disorders. Trends Neurosci 2004; 27 (7): 400—406.

19. Gonzalez-Burgos G, Lewis DA. GABA neurons and the mechanisms of network oscillations: implications for understanding cortical dysfunction in schizophrenia. Schizophr Bull 2008; 34 (5): 944-961.

20. Rudy B et al. Three Groups of Interneurons Account for Nearly $100 \%$ of Neocortical GABAergic Neurons. Dev Neurobiol 2010; 71: 45-16.
21. Runyan CA et al. Response features of parvalbumin-expressing interneurons suggest precise roles for subtypes of inhibition in visual cortex. Neuron 2010; 67 (5): 847-857.

22. Schiff ML, Reyes AD. Characterization of thalamocortical responses of regular-spiking and fast-spiking neurons of the mouse auditory cortex in vitro and in silico. J Neurophysiol 2012; 107 (5): 1476-1488.

23. Tan Z, Hu H, Huang ZJ, Agmon A. Robust but delayed thalamocortical activation of dendritic-targeting inhibitory interneurons. Proc Nat Acad Sci 2008; 105 (6): 2187-2192.

24. Busche MA et al. Decreased amyloid-beta and increased neuronal hyperactivity by immunotherapy in Alzheimer's models. Nature Neurosci 2015; 18 (12): 1725-1727.

25. Palop JJ, Mucke L. Network abnormalities and interneuron dysfunction in Alzheimer disease. Nature Rev Neurosci 2016; 7 (12): 777-792.

26. Verre, $\mathbf{L}$ et al. Inhibitory Interneuron Deficit Links Altered Network Activity and Cognitive Dysfunction in Alzheimer Model. Cell 2012; 149 (3): 708-721.

27. Xiao MF et al. NPTX2 and cognitive dysfunction in Alzheimer's Disease. Life 2017; 6: e23798.

28. Busche MA et al. Critical role of soluble amyloid- for early hippocampal hyperactivity in a mouse model of Alzheimer's disease. Proc Nat Acad Sci 2012; 109 (22): 8740-8745.

29. Grienberger $\mathbf{C}$ et al. Staged decline of neuronal function in vivo in an animal model of Alzheimer's disease. Nature Comm 2012; 3: 710-774.

30. Liebscher S et al. Selective Persistence of Sensorimotor Mismatch Signals in Visual Cortex of Behaving Alzheimer's Disease Mice. Curr Biol 2016; 26: 956-964.

31. Cacucci F et al. Place cell firing correlates with memory deficits and amyloid plaque burden in Tg2576 Alzheimer mouse model. Proc Nat Acad Sci 2008; 105 (22): 7863-7868.

32. Schmid LC et al. Dysfunction of Somatostatin-Positive Interneurons Associated with Memory Deficits in an Alzheimer's Disease Model. Neuron 2016; 92 (1): 114-125.

33. Siskova $\mathbf{Z}$ et al. Dendritic structural degeneration is functionally linked to cellular hyperexcitability in a mouse model of Alzheimer's disease. Neuron 2014; 84 (5): 1023-1033.

34. Roberson ED et al. Reducing endogenous tau ameliorates amyloid beta-induced deficits in an Alzheimer's disease mouse model. Science 2007; 316 (5825): 750-754.

35. Ittner LM et al. Dendritic Function of Tau Mediates Amyloid- b Toxicity in Alzheimer's Disease Mouse Models. Cell 2010; 142: 387-397.

36. Mairet-coello G et al. Article The CAMKK2-AMPK Kinase Pathway Mediates the Synaptotoxic Effects of A b Oligomers through Tau Phosphorylation. Neuron 2013; 78 (1): 94-108.

37. Kastanenka KV et al. Optogenetic Restoration of Disrupted Slow Oscillations Halts Amyloid Deposition and Restores Calcium Homeostasis in an Animal Model of Alzheimer's Disease. PLOS one 2017;12 (1): e0170275.

38. Iaccarino HF et al. Gamma frequency entrainment attenuates amyloid load and modifies microglia. Nature 2016; 540 (7632): 230-235.

39. Ke Y et al. Tau-Mediated Nuclear Depletion and Cytoplasmic Accumulation of SFPQ in Alzheimer's and Pick's Disease. PLOS one 2012; 7 (4): e35678. 
40. Zilka $\mathbf{N}$ et al. Truncated tau from sporadic Alzheimer's disease suffices to drive neurofibrillary degeneration in vivo. FEBS Lett 2006; 580: 3582-3588.

41. Santacruz K et al. Tau Suppression in a Neurodegenerative Mouse Model Improves Memory Function. Science 2005; 115: 476-482.

42. Ossenkoppele $\mathbf{R}$ et al. Tau PET patterns mirror clinical and neuroanatomical variability in Alzheimer's disease. Brain J Neurol 2016; 139 (Pt 5): 1551-1567.

43. Menkes-caspi $\mathbf{N}$ et al. Report Pathological Tau Disrupts Ongoing Network Activity. Neuron 85 (5): 959-966.

44. Jackson JS et al. Altered Synapse Stability in the Early Stages of Altered Synapse Stability in the Early Stages of Tauopathy. Cell Reports 2017; 18 (13): 3063-3068.

45. Ciupek SM et al. Progressive functional impairments of hippocampal neurons in a tauopathy mouse model. J Neurosci 2015; 35 (21): 8118-8131.

46. Booth CA et al. Altered Intrinsic Pyramidal Neuron Properties and Pathway-Specific Synaptic Dysfunction Underlie Aberrant Hippocampal Network Function in a Mouse Model of Tauopathy. J Neurosci 2016; 36 (2): 350-363

47. Booth CA et al. Electrical and Network Neuronal Properties Are Preferentially Disrupted in Dorsal, But Not Ventral, Medial Entorhinal Cortex in a Mouse Model of Tauopathy. J Neurosci 2016; 36 (2): 312-324.

48. Rocher AB et al. Structural and functional changes in tau mutant mice neurons are not linked to the presence of NFTs. Exp Neurol 2010; 223 (2): 385-393

49. Crimins JL et al. Homeostatic responses by surviving cortical pyramidal cells in neurodegenerative tauopathy. Acta Neuropathol 2011; 122 (5): 551-564.

50. Crimins JL, Rocher AB, Luebke JI. Electrophysiological changes precede morphological changes to frontal cortical pyramidal neurons in the rTg4510 mouse model of progressive tauopathy. Acta Neuropathol 2012; 124: 777-795.
51. Kopeikina KJ et al. Tau Causes Synapse Loss without Disrupting Calcium Homeostasis in the rTg4510 Model of Tauopathy. PLOS one 2013; 8 (11): e80834.

52. Rudinskiy $\mathbf{N}$ et al. Tau pathology does not affect experience-driven responses. Acta Neuropathol 2014; 2: 63.

53. Fu H et al. Tau Pathology Induces Excitatory Neuron Loss, Grid Cell Dysfunction, and Spatial Memory Deficits Reminiscent of Early Alzheimer's Disease. Neuron 2017; 93 (3): 533-541.e5.

54. Levenga $\mathbf{J}$ et al. Tau pathology induces loss of GABAergic interneurons leading to altered synaptic plasticity and behavioral impairments. Acta Neuropathol 2013; 1: 34.

55. Ahnaou A et al. Emergence of early alterations in network oscillations and functional connectivity in a tau seeding mouse model of Alzheimer's disease pathology. Sci Rep 2017; 7: 14189.

56. Jadhav S et al. Tau-mediated synaptic damage in Alzheimer's disease. Translat Neurosci 2015; 6 (1): 214-226.

57. DeKosky ST, Scheff SW. Synapse loss in frontal cortex biopsies in Alzheimer's disease: correlation with cognitive severity. Ann Neurol 1990; 27 (5): 457-464.

58. Braak H, Braak E. Neuropathological stageing of Alzheimer-related changes. Acta Neuropathol 1991; 82 (4): 239-259.

59. Wu JW et al. Neuronal activity enhances tau propagation and tau pathology in vivo. Nature Neurosci 2016; 19 (8): 1085-1092.

60. Pooler AM et al. Amyloid accelerates tau propagation and toxicity in a model of early Alzheimer's disease. Acta Neuropathol 2015; 3: 14.

61. Canter RG, Penney J, Tsai LH. The road to restoring neural circuits for the treatment of Alzheimer's disease. Nature 2016; 539: 187-196.

62. Schöll M et al. HHS. Publ Access 2017; 89 (5): 971-982.

63. Akwa Y et al. Synaptic activity protects against AD and FTD-like pathology via autophagic-lysosomal degradation. Mol Psychiat 2017. doi: 10.1038/mp.2017.142. 